\title{
INFLUENCIA DEL PATRÓN CULTURAL DIETÉTICO DEL PACIENTE DIABÉTICO EN EL APEGO AL RÉGIMEN ALIMENTICIO
}

Noemí Alcaraz Moreno*Ana María Chávez Acevedo**Genoveva Amador Fierros***Alma Cristina Reyes Alfaro****Jessica Isabel Loya Bayardo**** María

\section{Rocío del Carmen Vargas Ruelas****}

* Doctoranda- becaria por la Universidad de Colima, Programa Cultura de los

Cuidados. Universidad de Colima (UDC), México.

**MC. Profesora de la Facultad de Enfermería. UDC

***ME. Profesora de la Facultad de Enfermería. UDC

****Alumnas del $8^{\circ}$ semestre de la Carrera de Licenciatura en Enfermería. UDC.

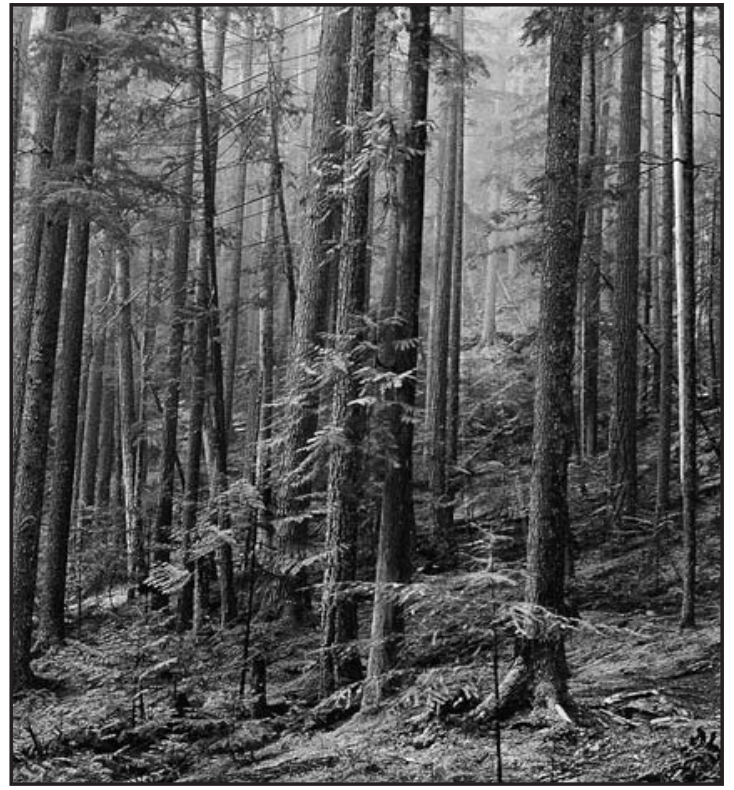

INFLUENCE OF DIABETIC PATIENTS' CULTURAL DIETETIC PATTERN ON THE ALIMENTARY ADHERENCE

\section{SUMMARY}

Introduction: Diabetic patients face the challenge of ensuring a new health status that leaves bad eating habits behind to adapt to new healthy habits. The road ahead is difficult, even when being fully aware that this is the ticket to a better quality of life.
Objective: To describe the influence that the cultural dietary pattern of diabetic patients has on the way they stick to their diet.

Methodology: This is a descriptive study in which the qualitative paradigm is used for both the design and information collection phases. Grounded theory is used to analyze data.

Conclusion: The cultural pattern has an influence when the patient faces a dietary change, sometimes to the extent that they make every effort to "reduce the intake, but not stop it." It is therefore necessary to ensure that the health care team monitor the disease, to implement individual educational and dietary programs and to involve the family in the process as a health care resource.

Key words: Cultural pattern; diet, diabetes; cultural health care.

INFLUÊNCIA DO PADRÃO CULTURAL DIETÉTICO DO PACIENTE DIABÉTICO NA ADESÃO AO REGIME ALIMENTAR

\section{RESUMO}

Introdução: Os pacientes diabéticos enfrentam o desafio de construir um novo estado de saúde, no qual têm que deixar para trás as más práticas alimentares, a fim de se acostumarem com novos hábitos saudáveis. A mudança não é fácil, mesmo havendo consciência de que essa é o meio para se obter uma melhoria da qualidade de vida. 
Objetivo: Descrever a influência do padrão cultural dietético do paciente diabético na adesão ao regime alimentar.

Metodologia: Trata-se de um estudo descritivo, no qual se faz uso do paradigma qualitativo, tanto para o desenho quanto para a coleta da informação; para tanto, utiliza-se a teoria fundamentada dos dados.

Conclusão: $O$ padrão cultural influi no momento em que a pessoa se depara com a mudança alimentar, em ocasiões em que se requer um esforço para "diminuir o consumo alimentar, sem eliminar eliminá-lo". É necessário o acompanhamento da equipe de saúde durante o trajeto de sua enfermidade, a implementação de programas educativos no tocante à alimentação de forma personalizada, bem como a incorporação da família a tais programas, como recurso de saúde (ou de sucesso no programa).

Palavras-chave: Padrão cultural; Regime dietético; Diabetes; Cuidados culturais.

\section{RESUMEN}

Introducción: Los pacientes diabéticos, se enfrentan al desafío de construir un nuevo estado de salud, en el que tienen que dejar atrás las malas prácticas alimenticias para acomodar nuevos hábitos saludables. El camino no es fácil aún cuando se tenga conciencia de que esa es la vía para tener una mejor calidad de vida.

Objetivo: Describir la influencia del patrón cultural dietético del paciente diabético en el apego al régimen alimenticio.

Metodología: Se trata de un estudio descriptivo, en el que se hace uso del paradigma cualitativo tanto para el diseño como para la recolección de la información y se utiliza la teoría fundamentada para el análisis de datos.

Conclusión: El patrón cultural influye al momento, en el que, la persona se enfrenta al cambio de alimentación, en ocasiones en tal grado que su esfuerzo se centra en "disminuir su consumo, no eliminarlo". Por lo que se hace necesario el acompañamiento del equipo de salud durante el trayecto de su enfermedad, la implementación de progra- mas educativos y alimenticios personalizados, así como la incorporación de la familia como recurso de salud.

Palabras Clave: Patrón cultural; régimen dietético, diabetes; cuidados culturales.

\section{INTRODUCCIÓN}

Numerosos estudios, fundamentan la importancia de una alimentación saludable y balanceada para la prevención y tratamiento de la diabetes mellitus. En muchos casos el seguimiento adecuado de un régimen alimenticio resulta suficiente para controlar dicha patología, sin embargo la cultura puede actuar como una barrera que impide a un buen número de pacientes apegarse al régimen.

El individuo y su familia se conducen dentro de una cultura alimentaria propia, construida a partir de emociones, ideas, significados, alimentos y prácticas alimentarías de la cual todos ellos se sienten parte. A su vez, esta cultura alimentaria es compartida por toda una comunidad y se va reintegrando de una generación a otra. Por lo tanto, las personas diabéticas se encuentran al centro de un proceso de transformación cultural alimentaría más amplio (Castro S. A. 2007) y les es difícil asimilar un cambio radical impuesto a un estilo de vida arraigado a su herencia cultural. El desafío de construir un nuevo estado de salud en el que se tienen que dejar atrás las malas prácticas alimenticias para acomodar nuevos hábitos saludables no es un camino fácil aún cuando se tenga conciencia de que esa es la vía para tener una mejor calidad de vida.

La cultura es pues fundamental para el tema que nos ocupa. Su concepto ha ocupado a múltiples pensadores de los que derivan al menos entre 150 y 200 definiciones, (Lipson, 2000), lo que puede dar una idea de la complejidad del terreno que pisamos cuando se integra esta variable en los análisis de salud; no obstante, para este estudio retomamos el concepto de Malinowski (en Firth, R; et al. 1997), quien considera que cultura es un equivalente de "herencia social o conjunto de fuerzas que inciden en el individuo nacido en una sociedad determinada", dentro de ella incluye: los bienes, los procesos técnicos, las ideas, los hábitos y los valores. Dicho concepto invita a mirar hacia 
la antropología para comprender desde ahí los significados que el paciente y su familia otorgan a la enfermedad, con la certidumbre de que no siempre coinciden con el significado construido para ello por el equipo de salud. (Amezcua, 2000).

Aún desde esas miradas distintas, la enfermería y la antropología, comparten rasgos característicos, (Siles, 2003) como: su campo de conocimientos referidos al ser humano, a través de la triada: individuo, familia y comunidad; sus herramientas metodológicas, al ocuparse del estudio de la satisfacción de necesidades de la comunidad desde la perspectiva de los cuidados y la influencia de los factores culturales en dicho proceso, así como su praxis, encaminada a "adaptar los cuidados a la idiosincrasia de la cultura del individuo, la familia o la comunidad".

La competencia cultural ha sido identificada como inherente al quehacer de la enfermería y por ello, se le ha instado a superar retos relacionados con las prácticas religiosas y la diversidad en la forma de satisfacer las necesidades dentro de los diferentes grupos sociales, Siles (2003). Para afrontarlos, la enfermería cuenta con aportaciones realizadas desde el interior de la disciplina a través de modelos y teorías, como la de Madeleine Leininger con su teoría "Diversidad y universalidad en el cuidado cultural" y su modelo de "el sol naciente". Ambos derivan de la enfermería transcultural, definida por la autora como "área formal de estudio y trabajo centrado en el cuidado basado en la cultura, creencias de salud o enfermedad, valores y prácticas de las personas, para ayudarlas a mantener o recuperar su salud, hacer frente a sus discapacidades o a su muerte (Leininger, 1978, 1995, citado en Leininger. 1999). El trabajo de Rachel Spector, describe "los Cuidados Culturales y las formas de identificar las creencias, prácticas y tradiciones de salud de pacientes y familias que son miembros de cualquier grupo cultural" (Spector, R. 1999), mientras que el modelo de competencia cultural de Purnell, tiene como objetivo "promover la comprensión cultural de situaciones humanas durante periodos de enfermedades, y el mantenimiento y promoción de la salud" (Purnell, L. 1999).

Con este bagaje de conocimientos y herramientas metodológicas, emprendimos el desafío de entender mejor la influencia de los patrones cultu- rales en el modo de actuar de los pacientes que han sido llamados a implantar cambios significativos en su vida, específicamente cambios en el régimen dietético, como un medio indispensable para ganar calidad de vida.

\section{OBJETIVO}

El objetivo general del estudio fue: describir la influencia del patrón cultural dietético del paciente diabético en el apego al régimen alimenticio. Para el logro de dicho objetivo nos planteamos tres objetivos específicos: conocer el patrón cultural dietético del paciente diabético; identificar el régimen alimenticio actual del paciente diabético y conocer la experiencia de asimilación y acomodación del régimen alimenticio del paciente diabético.

\section{ESTADO DE LA CUESTIÓN}

Podemos resaltar tres nociones, resultantes de la búsqueda de información relacionada con nuestro estudio, la primera de ellas expresa la falta de apego al tratamiento en general por parte de los pacientes diabéticos. Un estudio realizado por Durán, et al (2001), en 150 pacientes con diabetes, reveló que el apego correspondió a 54.2\%, los factores asociados al no apego fueron la escolaridad primaria y la falta de información sobre la enfermedad. Por su parte Hernández (2003), en una población de 79 diabéticos, midió el grado de cumplimiento para cada una de las medidas terapéuticas que correspondía a: ingesta de medicamentos y/o insulinoterapia, dieta, ejercicio y asistencia a citas médicas, encontrando que el incumplimiento global fue de $39 \%$ y que tanto la dieta como el ejercicio eran las medidas en las que más se incumplía con un $62 \%$ y un $85 \%$ respectivamente. Con un estudio especifico del cumplimento de una dieta hipocalórica en un grupo de pacientes con diabetes tipo 2, (Román DL, Izaola O, Aller, 2001), identificaron que los pacientes realizan una dieta hipocalórica, con predominio de hidratos de carbono, alto porcentaje de grasas monoinsaturadas y un aporte de proteínas correcto.

No obstante, se considera que un programa integral que incluya actividades educativas teórico-prácticas en los aspectos medico, nutricional, social y psicológico, mejorará considerablemente las condiciones generales de estos pacientes, lo 
cual fue demostrado en los estudios realizados por Jauregui (2002), Amezcua (2002) y Castro (2007).

Cuando se considera a los hábitos alimenticios parte de una cultura y no como sólo como componente de medidas terapéuticas para un determinado padecimiento (como lo concibe el paradigma biologicista médico), plantea la necesidad de situarnos dentro de la perspectiva antropológica para comprender el significado que tiene para las personas que padecen diabetes, el concepto de dieta (Mendoza, et al. 2000), al mismo tiempo que se aprende a respetar y aceptar las diferencias culturales (Vásquez, M. 2000; (Rohrbach, 1998), evitando así el choque cultural que con frecuencia se establece entre pacientes y equipo de salud (Amezcual, 2000).

\section{METODOLOGÍA \\ Diseño}

Se trata de un estudio descriptivo, en el que se hace uso del paradigma cualitativo tanto para el diseño como para la recolección de la información y se utiliza la teoría fundamentada para el análisis de datos. La investigación cualitativa permite al investigador situarse directamente con los actores y dentro del contexto de un fenómeno determinado, estudiar la cultura en uno de sus rasgos característicos como es el caso de la alimentación. Este enfoque nos obliga literalmente a indagar dentro del espacio personal tanto como del contextual en un grupo de personas. Por su parte, la teoría fundamentada, tiene como objetivo final explicar la realidad que se está investigando, a través de la recolección e interpretación de los datos, apoyados en explicaciones teóricas (Trinidad, A; Carrero, V y Soriano, R, 2006).

\section{Recolección de datos}

El trabajo de campo se llevó a cabo entre los meses de junio a septiembre del 2008. Para la recolección de la información se empleó la entrevista semi-estructurada, ya que, es la que permite que los sujetos entrevistados expresen de una manera más abierta sus puntos de vista, su uso nos permitió tomar decisiones respecto a la secuencia de preguntas y conducir al informante cuando se requirió profundizar en alguna temática determina (Flick, 2004).
Previo consentimiento informado, se aplicaron 10 entrevistas a personas que padecen diabetes tipo 2, adscritas a una Institución de segundo nivel de atención, perteneciente a la seguridad social, del municipio de Colima, México. De ellos, 5 son mujeres y 5 hombres, pertenecen a zonas urbanas de los municipios de Colima y Villa de Álvarez; es un grupo heterogéneo respecto a su edad, la cual oscila entre los 86 años del paciente más longevo hasta 32 años de la persona con menos edad dentro del grupo.

El análisis de datos se hizo de forma manual, el proceso llevado a cabo fue el siguiente: se transcribieron cada una de las entrevistas, se aplicó el microanálisis, que consiste en analizar la información línea por línea (Strauss, A; Corbin, J.2002), identificando aquellos datos que a nuestro juicio fueron importantes para nuestro objetivo. La información obtenida del microanálisis se clasificó en fichas, las que contienen: 1) datos de la persona entrevistada, 2) párrafo de entrevista 3) memos analíticos y 3) el código que representa el dato identificado. Una vez obtenidos los códigos se definieron las categorías, sus dimensiones y subcategorías, para luego contrastarlas con teorías precedentes.

\section{RESULTADOS}

A continuación se presenta el desarrollo y la discusión de las dos categorías resultantes del presente trabajo: la primera de ella "la experiencia de confrontar hábitos alimenticios no saludables", da cuenta de la realidad alimentaria de la que parten los pacientes al momento en que se les informa el diagnóstico de su enfermedad y la difícil experiencia por la que pasan al afrontar la indicación de su cambio de hábitos alimenticios. La segunda categoría "educación para la salud", refleja la necesidad de proporcionar una adecuada educación de salud por parte del equipo que interviene en el cuidado de las personas con esta patología.

\section{Categoría: la experiencia de confrontar hábitos alimenticios no saludables.}

Como sabemos las enfermedades llamadas crónicas, grupo al que pertenece la enfermedad de nuestros informantes, causan un gran impacto en la vida de las personas. Según Brannon y Feist (2001), 
dos situaciones conducen a éste desequilibrio: primero "la reacción emocional" que produce el saberse portador de una enfermedad de por vida y la segunda por los cambios que deberá realizar la persona en su "estilo de vida". Del mismo modo, el patrón cultural es un aspecto condicionante para que los pacientes con diabetes sigan su tratamiento alimenticio (Smeltzer, SC; Bare BG. 2005).

Estas prácticas, que se fundamentan en la cultura adquieren particular importancia en el cuidado de pacientes con Diabetes, "para quienes la dieta desempeña un papel crucial en su tratamiento y para la conservación de su salud" (Cardoso. 2006). En nuestro caso, los informantes refirieron que para ellos, lo más "difícil de llevar es la dieta" ya que deben cambiar hábitos que tienen desde hace mucho tiempo, destacando además el carácter súbito del cambio. Sus respuestas reflejan que no han modificado sus hábitos alimenticios. La mayoría comenta que lo que han hecho es disminuir las cantidades o variar la frecuencia de su consumo, incluso hay quienes aseguran "sigo con el mismo ritmo de vida, pues no puede dejar uno nada, porque es parte de la vida de uno".

Identificamos, que existen diversos hábitos relacionados con el patrón cultural, que se consideran limitante para modificar sus prácticas alimenticias. Entre ellos, destacan las reuniones familiares y/o el comer fuera de casa, en especial los fines de semana, los festejos con motivo de cumpleaños, navidad y año nuevo, en donde suele prevalecer la comida típica de la localidad o de la región, cuyas materias primas son una gran fuente de grasas, carbohidratos y azúcares" (Oseguera. 1995). Mencionan también que hay sentimientos de por medio que les impiden cumplir con la dieta debido a que extrañan la alimentación que llevaban antes de su enfermedad. Ellos relacionan el régimen con "sacrificio o con imposición" e incluso refieren que se sienten tristes al no poder comer sus comidas favoritas.

Un aspecto primordial, para lograr el apego de los pacientes al régimen alimenticio es sin duda el apoyo que reciban de sus familias. Es precisamente este aspecto que consideramos una tarea pendiente de los servicios de salud. Incorporar a la familia como apoyo a su familiar con diabetes es de fundamental importancia. De acuerdo con la tipología establecida por Castro (2007), para identificar los patrones de acomodación socio alimentaría que llevan a cabo las familias con pacientes diabéticos, ubicaríamos a nuestros informantes en la familia segregante, esto significa que la familia aparta o separa a la persona diabética, en especial en lo que refiere al rubro de alimentación. Incluso algunas personas se sienten culpables de tener la enfermedad, por lo que no realizan ningún cambio en su alimentación para no sacrificar al resto de la familia, en especial a los hijos.

Otra de las limitantes, está relacionada con las creencias y/o mitos de las personas respecto a la diabetes. La mayoría de ellos consideran que lo que más "hace daño" es el consumo de azúcares, por lo que refieren que han disminuido el consumo de refresco o lo han cambiado por refrescos hechos a base de edulcorantes artificiales, sin embargo desconocen la importancia del manejo de los carbohidratos para el control de su enfermedad (Lerman, 2003).

Además del patrón cultural, se encontraron otros limitantes para el apego al régimen alimenticio de las personas que padecen diabetes, los cuales están determinados por su actividad laboral y el rol familiar y social desempeñado.

\section{Categoría: Educación para la salud}

La educación entendida como un proceso de enseñanza-aprendizaje que permite adquirir conocimientos para resolver problemas (Islas S.A. 2005) nos hace suponer que cuando la persona adquiere e integra un nuevo conocimiento o experiencia en su vida, se esperaría que ello lo lleve a modificar total o parcialmente su comportamiento, es decir, se esperaría que aprenda. La educación que reciben las personas con diabetes está pensada, desde los servicios de salud, con esa lógica. Es decir, se espera como resultado de la acción educativa, que los pacientes modifiquen sus hábitos alimenticios y el apego al régimen alimenticio prescrito por los profesionales de salud. Nada más alejado de la realidad mientras no se tome en cuenta la variable cultural. Todos nuestros informantes tienen más de un año de padecer la enfermedad (la antigüedad oscila entre 1 a 32 años) y se encuentran en seguimiento médico y aún así, no han conseguido llevar el régimen alimenticio prescrito. 
Los informantes perciben la dieta como un ideal casi imposible de realizar, se le conoce como "dieta rigurosa" y se le identifica como "la lista, hoja o papel de la nutrióloga", esto en referencia al plan nutricional estandarizado (despersonalizado) que los pacientes reciben por escrito luego de la consulta de nutrición.

Se encontró que las dietas son otorgadas por el médico familiar, el internista o la trabajadora social y no por una nutrióloga como sería deseable. Además se entrega una misma dieta a todos los pacientes siendo que "El plan de alimentación de los diabéticos debe tomar en consideración los tipos de alimentos que agradan al paciente, su estilo de vida, la hora en que normalmente comen y los antecedentes étnicos y culturales" (Smeltzer, Bare, 2005). En la actualidad se considera que en lugar de hacer énfasis en el porcentaje calórico que debe obtenerse de nutrimentos específicos, hay que enfocarse en los alimentos que consume el paciente, los cambios que pudieran ser benéficos y los cambios que esté dispuesto o sea capaz de lograr, puesto que al final es la persona quien decide que alimentos consumir.

\section{DISCUSIÓN Y CONCLUSIONES}

Consideramos que el patrón cultural, influye al momento en que la persona se enfrenta al cambio de alimentación, ya que la alimentación va más allá de la ingesta de alimentos, representa tradiciones, valores, creencias, acumulados a lo largo de la vida y que forma parte de la subjetividad de cada ser humano.

La dificultad para seguir el régimen alimenticio establecido por los profesionales de salud, radica en que hay sentimientos de por medio que actúan como una barrera: 1) Se extraña la alimentación que llevaban antes de su enfermedad, 2) el cumplir la dieta representa un sacrificio o una imposición, 3) no logran visualizar la importancia que que la dieta representa para su salud.

Por lo anterior consideramos que en el control y seguimiento de los pacientes con esta enfermedad, el equipo de salud debería hacer énfasis en los siguientes aspectos: brindar apoyo psicológico durante la etapa de aceptación de la enfermedad y de modificación de su estilo de vida; establecer programas educativos con base en las característi- cas personales y culturales de cada uno de los pacientes; elaborar dietas personalizadas de acuerdo a la edad, sexo y cultura alimenticia, de tal manera que el cambio no represente para el/la paciente "un choque cultural".

El establecimiento de las estrategias expuestas, exige un trabajo conjunto y planificado del equipo multidisciplinario, al mismo tiempo que cada profesional lleva a cabo sus funciones específicas. En ese sentido identificamos la ausencia del personal de enfermería, pues en ningún momento se observó su participación dentro del grupo de apoyo, por lo que sugerimos se involucre a esta disciplina tanto en la educación de los pacientes como en el control y seguimiento de su dieta con miras a que los pacientes se sientan acompañados durante la experiencia de modificar sus hábitos alimenticios.

La incorporación de la familia como componente indispensable del cuidado y en especial del acompañamiento al paciente en el apego al régimen dietético resulta de fundamental importancia. La educación a la comunidad respecto a la importancia del apego al régimen dietético como un medio para conseguir una mejor calidad de vida de los enfermos de diabetes y como medio para una vida saludable en sus familiares, resulta crucial. Finalmente consideramos que hacen falta esfuerzos de investigación dirigidos a conocer más sobre los aspectos subjetivos de la enfermedad que determinan la forma de actuar, más que la forma de pensar de los pacientes. De este tipo de medidas dependerá que se proporcionen "cuidados culturalmente adecuados".

\section{BIBLIOGRAFÍA}

- Amezcua, RC. et al. (2002) Tratamiento en el paciente diabético. HGZ57 "La Quebrada". sep-dic; Vol. I (1): p 20 - 23. Consultado en la red mundial el 12 de abril de 2008 en: http://www.medigraphic.com/espanol/e-htms/e-quebrada/elu2002/elq02-1/em-lq021e.htm

- Amezcua, M. (2000) Antropología de los Cuidados, Enfermedad y Padecimiento: Significado de Enfermar para la Práctica de los Cuidados. Rev. Cultura de los Cuidados. $1^{\circ}$ y $2^{\circ}$ semestres. Año IV. No. 7 y 8.

- Brannon, L; Feist, J. (2001) Psicología de la Salud. España:Paraninfo/Thomson Learning.

- Cardoso, GMA. (2006) La cultura como configurador de estilos alimentarios antagónicos al tratamiento médico contra la diabetes, cultura, dieta y diabetes. Redalyc may-ago; Vol. 13 (32): p 129 - 147. Consultada en la red mundial el 10 de marzo de 2008 en: http:/redalyc.uaemex.mx/redalyc/pdf/351/ 35103707.pdf 
Castro, S.A. (2007) La nutrición como ruptura cultural: la experiencia de los adultos con diabetes mellitus tipo 2 . Investigación en Salud. Abr. 1; Vol. IX (1): p 26 - 33. Obtenida de la red mundial el 27 de febrero de 2008. Consultada http://web.ebscohost.com/ehost/pdf?vid=5\&hid=2\&sid=1 af9 bc55-07e8-4dfe-adcd-132c92f7defb\%40sessionmgr7

Duran; Varela B. (2001) Apego al tratamiento farmacológico en pacientes con diagnóstico de diabetes mellitus tipo 2. Salud Pública de México. May-jun. Vol. 43. (3): p. 233-236. Consultado en la red mundial el 12 de abril de 2008 en: http://scielo.unam.mx/pdf/spm/v43n3/a09v43n3.pdf

Firth, R, et al. (1997) Hombre y Cultura. La obra de Bronislaow Malinoswki. España: Siglo Veintiuno.

Flick, U. (2004) Introducción a la investigación cualitaiva. Madrid, España: Morata

Hernandez, 1. (2003) Factors associated with therapy noncompliance in type-2 diabetes patients. Salud pública de México. May-jun, 45, 191-197. Consultado en la red mundial el 4 de marzo de 2008: http://www.scielosp.org/pdf/spm/v45n3/ 16481.pdf

Islas, AS. (2005) Diabetes Mellitus, $3^{\mathrm{a}}$ ed. México, Mc GrawHill/ Interamericana, p 381, 421-423

- Jáuregui, J.J; De la Torre S.A; Gómez G.P. (2002) Control del padecimiento en pacientes con diabetes tipo 2 e hipertensión arterial: impacto de un programa multidisciplinario. Revista Médica del IMSS. Ene. 24; Vol 40 (4): p. 307-318. Consultado en la red mundial el 10 de marzo de 2008 en: http://www.medigraphic.com/pdfs/imss/im-2002/im024f.pdf Leininger, M. (1999) Cuidar a los que son de culturas diferentes requiere el conocimiento y las aptitudes de la enfermería transcultural.Cul Cuid. 1999 jul-dic;Año III(6):5-8.

Lerman, I. (2003) Atención integral del paciente diabético 2a ed. México: McGraw-Hill Interamericana Editores, p 55, 8183

Lipson, JG. (2000) Cultura y Cuidados de Enfermería. Index de Enfermería [Index Enferm] (edición digital) 2000; 28-29. Disponible en <http://www.index-f.com/index-enfermeria/2829revista/28-29_articulo_19-25.php> Consultado el 29 de Mayo de 2008.

Mendoza, V.F. et al. (2000) Comunicación y perspectiva de audiencias: una investigación cualitativa para la diabetes. Revista de endocrinología y nutrición. Ene-mar; Vol 8 (1): p. 5-13. Consultado en la red mundial el 12 de abril de 2008 en: http://www.medigraphic.com/pdfs/endoc/er-2000/er001b.pdf Oseguera, D. (1995). La cocina colimense, el menú muestrario de la cultura regional. Estudios sobre las culturas contemporáneas. Dic.1995/Vol.1, número 002. Universidad de Colima, Colima, México. En http:// redalyc.uaemex.mx

Purnell, L. (1999) El modelo de competencia cultural de Purnell: descripción y uso en la práctica, educación, administración e investigación.Cul Cuid. 1999 jul-dic;Año III(6):9197.

Rohrbach; Viadas, C. (1997). Antropología: cuidar es tan antiguo como el mundo y tan cultural como la diversidad de la humanidad Cultura de los cuidados, cultura de los cuidados. No. 2: 36-39. Consultada en la red mundial el 13 de mayo de 2008 En: http://www.index-f.com/cultura/revista.php

Román, D.L; Izaola, O; Aller, R. (2001) Evaluación del cumplimiento de una dieta de 1.500 calorías en una población de diabéticos tipo 2 con sobrepeso. Nutrición hospitalaria. Vol.
16 (4); p. 122-125. Consultado en la red mundial el 14 de abril de 2008 en: http://www.grupoaulamedica.com/web/nutricion/pdf/042001/eva1.pdf

- Siles, J. (2003) Antropología y Enfermería. La necesaria simbiosis entre dos disciplinas para vertebrar culturalmente la teoría y la praxis de los cuidados. Index de Enfermería [Index Enferm] (edición digital) 43. Disponible en: $<$ http://www.index-f.com/index-enfermeria/43revista/43_articulo_28-32.php> Consultado el 30 de Mayo de 2008.

- Smeltzer, SC; Bare B.G. (2005) Enfermería Medicoquirúrgica, 10a ed México: McGraw-Hill; p 111-115, 128-131, 1279, 1289-1291 (Vol. 1).

- Spector. R. (1999). Cultura, cuidados de salud y enfermería.Cul Cuid. 1999 jul-dic;Año III(6):66-73.

- Strauss, A; Corbin, J. (2002) Bases de la investigación cualitativa: técnicas y procedimientos para desarrollar la teoría fundamentada. Medellín: Colombia.

- Trinidad, A; Carrero, V, Soriano, R. (2006). Cuadernos Metodológicos: Teoría fundamentada "grounded theory". España. Centro de investigaciones sociológicas. p 24-25.

- Vásquez, T.M. (2006) Aproximaciones a la creación de competencias culturales para el cuidado de la vida. Educación/Education. Sep. Vol. 24 (2); p. 136-142. Consultado en la red mundial el 14 de mayo de 2008 en: http://enfermeria.udea.edu.co/revista/v24n2/pdf/v24n2a15.pdf - Vásquez, T.M. (2000). El cuidado en enfermería desde la perspectiva transcultural: una necesidad en un mundo cambiante. Vol. 4 (3). Consultado en la red mundial el 14 de mayo de 2008 en: http://enfermeria.udea.edu.co/revista/v4n/pdf/v4n3.pdf

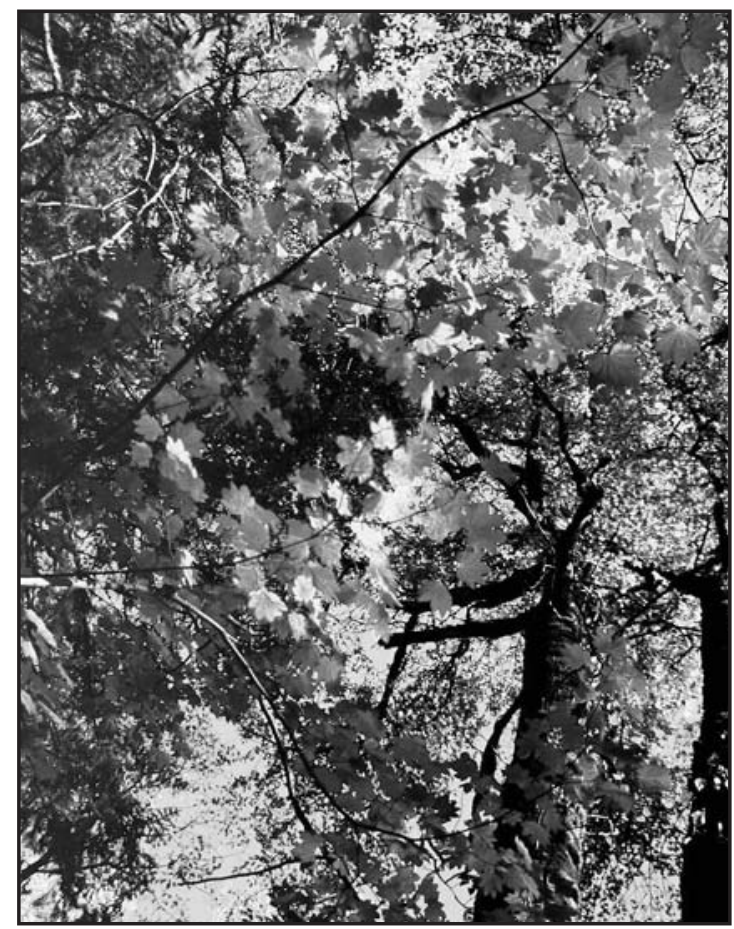

